\title{
Study on Marshall Stability Properties of BC Mix Used In Road Construction by Adding Waste Plastic Bottles
}

\author{
Shiva Prasad K, Manjunath K. R, K. V R Prasad
}

\subsection{Background}

\section{Introduction}

Due to increasing demand in highway construction, scientists and researchers are constantly trying to improve the performance of bitumen pavement. Asphalt concretes are widely used in pavements. Bitumen is the naturally occurring byproduct of crude oil. Due to increase in vehicles in recent years the road surfaces have been exposed to high traffic resulting in deformation of pavements due to excessive stress. Permanent deformation happens when pavement does not have sufficient stability, improper compaction and insufficient pavement strength.

The performance of pavement is determined by the properties of bitumen. Bitumen is a viscoelastic material with suitable mechanical and rheological properties for water proofing and protective covering for roofs and roads, because of its good adhesion properties of aggregates. One of the most important properties of bitumen mixture is its ability to resist shoving and rutting under traffic. Therefore stability should be high enough to handle traffic adequately, but not higher than the traffic conditions require. Low stability causes unraveling and flow of the road surface. Some improvements in asphalt properties have been achieved by selecting the proper starting crude, to make asphalt.

From practical experiences it is proved that the modification of asphalt binder with polymer additives, offers several benefits. To enhance various engineering properties of asphalt many modifiers such as styrene based polymers, polyethylene based polymers, polychloroprene, gilsonite, various oils have been used in asphalt.

Plastic usage has been increased in our daily life. Due to this increased usage of plastic the disposal of plastic has been difficult. Some studies say that 10million tones of plastic are produced in India and only 2 million tones of plastic waste are recycled. Plastics have to be disposed or else it will be hazardous to nature and environment. Thus one of the best ways of disposal of these plastics is to use in bituminous road construction by melting them. Many highway agencies are doing various studies on environmental suitability and performance of recycled products in high construction. Use of these waste plastic in bituminous road construction will help in disposal of vast quantities of plastic. Consumption of mineral water bottles which are made up of high density polyethylene has increased abnormally. These bottles are not readily biodegradable, environmental problems are created due to dumping; these are either land filled or incinerated which are not ecofriendly which pollute land and air.

\subsection{Objectives}

- To study basic properties of aggregates and plain bitumen.

- To study the strength and stability characters of BC mix for $60 / 70$ and 80/100 grade bitumen.

- To study the effect of waste plastic on strength and stability characteristics of BC mix.

1.3 Scope

An experimental work has been proposed to improve the properties of pavement using waste plastic

- Waste plastic are ground and mixed with hot bitumen and polymer modified binder is prepared.

- Laboratory studies will be carried out on polymer modified asphalt mixtures to evaluate engineering properties using marshal stability and indirect tensile strength.

\section{Literature review}

For many years, researchers and development chemists have experimented with modified bitumen mainly for industrial uses, adding asbestos, special filler, mineral fibers and rubber. In the last thirty years many researchers have looked at a wide spectrum of modifying materials for bitumen's used in road construction.

The study was done by Mahabir Panda ${ }^{[1]}$ and Mayajit Mazumdar using 80/100 penetration grade bitumen and Ethylene Vinyl Acetate (EVA) copolymer. The study that there was a increase in stability value in case of polymer modified bitumen. Stability value was high as $14 \mathrm{kN}$ in case of polymer modified bitumen. Tensile strength was also increased and stripping properties were improved.

Another study was done by Sharma D K ${ }^{[2]}$ and others using 60/70 penetration grade bitumen. Here waste plastic/polymer was used as modifiers. The waste plastic/polymer was added on the aggregate before 
Study on Marshall Stability Properties of BC Mix Used In Road Construction by Adding Waste mixing Optimum Binder Content (OBC) in dry process at $150-160^{\circ} \mathrm{C}$ temperature. This type of mixing increases the bonding between aggregates coated with plastic/polymer which increases the strength of the bituminous concrete mixes. Stability values and indirect tensile strength values were observed to be more in polymer modified bitumen than in conventional bitumen. Rutting values were also higher in polymer modified bitumen mixes than in conventional mixes.

Another study was carried out by Shivangi Gupta and Veeraragavan ${ }^{[3]}$. They used 60/70 penetration grade bitumen and Styrene Butadiene Styrene (SBS) modified binder. Here tests were conducted by two methods, marshal stability and Superpave Gyratory Compactor (SGC) and results of these two methods were compared. The test results showed that SBS modified bitumen mixes were superior to the conventional mixes. But as far as Marshall Method is concerned SGC method shows better results. Strength parameters like tensile strength, marshal stability values of SBS modified mixes were higher than $21 \%$ to $25 \%$ than that of conventional mixes. Fatigue life of SBS modified binder mix was $2.1 \%$ to $2.4 \%$ higher than the conventional mix.

\section{Present investigation}

Present investigation of the work is to investigate the effects of waste plastic bottles on the strength and stability characteristics of $\mathrm{BC}$ mix which is used for surface course in road construction. In present investigation bituminous concrete $[\mathrm{BC}]$ of grade I is selected.

\subsection{Methodology Adopted For Present Study}

- To conduct the Standard tests for the properties of plain bitumen.

- To determine the optimum binder content for plain mixes 80/100,60/70, by Marshall Stability method.

- To use waste plastic as additive with aggregate and blended with bitumen and test all the basic test parameters.

\subsection{Material Characterization}

Study involves the use of materials like Bitumen, Aggregate and Polyethylene Terapthalate (PET)

\section{Bitumen}

Bitumen is a material which is a byproduct of petroleum refining process. It is a highly viscous at temperature above 100 degrees Celsius and is solid at room temperature.

Table 3.1: Properties of bitumen used in present study

\begin{tabular}{|c|c|c|c|c|}
\hline S1 No. & Properties & \multicolumn{2}{|c|}{ Grade } & \multirow{2}{*}{ Test methods } \\
\cline { 3 - 4 } & & $60 / 70$ & $80 / 100$ & \\
\hline 1 & Penetration at $25^{\circ} \mathrm{C}$ & 67 & 90 & IS:1203-1978 \\
\hline 2 & Softening point $(\mathrm{R} \& \mathrm{~B}){ }^{\circ} \mathrm{C}$ & 51 & 41 & IS:1205-1978 \\
\hline 3 & Ductility @ $27^{\circ} \mathrm{C}, \mathrm{cm}$ & 73.5 & 75.5 & IS:1208-1979 \\
\hline 4 & Flash point, ${ }^{\circ} \mathrm{C}$ & 330 & 261 & IS:1209-1981 \\
\hline 5 & Fire point, ${ }^{\circ} \mathrm{C}$ & 345 & 283 & IS:1209-1981 \\
\hline 6 & Specific gravity of bitumen & 1.017 & 1.02 & IS:1202-1980 \\
\hline
\end{tabular}

\section{Aggregates}

An aggregate which has good and sufficient strength, hardness, toughness and soundness have to be chosen. Crushed aggregates produce higher stability.

Basic physical parameters of aggregates are found using various tests as tabulated below.

Table 3.2: Properties of Aggregates used in present study

\begin{tabular}{|c|c|c|c|}
\hline Sl no & Aggregate tests & $\begin{array}{c}\text { Test results } \\
\text { obtained }\end{array}$ & $\begin{array}{c}\text { Requirements as per Table } \\
\text { 500-14 of MoRTH (IV } \\
\text { revision) Specifications }\end{array}$ \\
\hline 1 & Crushing value (\%) & 24.8 & - \\
\hline 2 & Impact value (\%) & 20.8 & Max 24\% \\
\hline 3 & Los Angeles abrasion value (\%) & 32 & Max 30\% \\
\hline 4 & Combined index (\%) & $29 \%$ & Max 30\% \\
\hline 5 & Water absorption (\%) & 0.25 & \multirow{2}{*}{$2.5-3.0$} \\
\hline 6 & Specific gravity of coarse aggregates & 2.72 & \\
\hline 7 & Specific gravity of fine aggregates & 2.76 & \\
\hline 8 & Specific gravity of filler & 2.5 & \\
\hline
\end{tabular}


Study on Marshall Stability Properties of BC Mix Used In Road Construction by Adding Waste 3. Plastic

Polyethylene Terapthalate (PET) is the type of plastic labeled with the \#1 code on or near the bottom of bottles and containers and is commonly used to package soft drinks, water, juice, peanut butter, bakery goods, produce, frozen foods, salad dressings and oil, cosmetics and household cleaner and many other products. Waste bottle plastic of water cans is made up of either High Density Polyethylene (HDPE) or Low Density Polyethylene (LDPE). These plastic bottles are shredded and used for the present investigation. followed

Waste plastic bottles were crushed and shredded. The physical properties of waste bottle plastic are as

Table 3.3: Properties of plastic used in present study

\begin{tabular}{|l|l|}
\hline Properties & Results obtained \\
\hline Specific gravity & 1.03 \\
\hline Melting point ${ }^{\circ} \mathrm{C}$ & $250-260$ \\
\hline Sieve analysis & Passing $4.75 \mathrm{~mm}$ sieve retained on $2.36 \mathrm{~mm}$ sieve \\
\hline
\end{tabular}

[Source: pheonixtechnologies.net]

\subsection{Selection of Aggregate gradation}

Selection of proper gradation for the mix is one of the most important parameter. Ministry of road transport $[\mathrm{MoRTH}]$ has given some of the grading specifications for all the bituminous and non-bituminous layers used in road construction. In this investigation bituminous concrete [BC] which is considered as the wearing course or the surface course is selected. There are two gradations for bituminous concrete layers Grade I and grade II. As per Ministry Of Road Transport [MoRTH] specification bituminous concrete of mix designation Grade I, nominal aggregate size of $19 \mathrm{~mm}$ and layer thickness of $50-60 \mathrm{~mm}$ is selected. Grading specification for bituminous concrete course is given in table 4.1

Table 3.4 Gradation of aggregates for Bituminous Concrete Pavement layers (BC)

\begin{tabular}{|c|c|c|}
\hline $\begin{array}{c}\text { Mix designation } \\
\text { Nominal aggregate size } \\
\text { Layer thickness }\end{array}$ & $\begin{array}{c}\text { Grading } 1 \\
19 \mathrm{~mm} \\
50-60 \mathrm{~mm}\end{array}$ & $\begin{array}{c}\text { Grading } 2 \\
13 \mathrm{~mm} \\
30-45 \mathrm{~mm}\end{array}$ \\
\hline IS sieve(mm) & Cumulative \% by weight of total aggregate passing \\
\hline 45 & - & - \\
\hline 37.5 & - & - \\
\hline 26.5 & 100 & - \\
\hline 19 & $79-100$ & $79-100$ \\
\hline 13.2 & $59-79$ & $70-80$ \\
\hline 9.5 & $52-72$ & $53-71$ \\
\hline 4.75 & $35-55$ & $42-58$ \\
\hline 2.36 & $28-44$ & $34-48$ \\
\hline 1.18 & $20-34$ & $26-38$ \\
\hline 0.6 & $15-27$ & $18-28$ \\
\hline 0.3 & $10-20$ & $12-20$ \\
\hline 0.15 & $5-13$ & $4-10$ \\
\hline 0.075 & $2-8$ & $5.0-7.0$ \\
\hline Bitumen content, \% by weight of & $5.0-6.0$ & 65 \\
\hline total mixture & & 65 \\
\hline Bitumen grade & &
\end{tabular}

Aggregates of size $25 \mathrm{~mm}, 12 \mathrm{~mm}, 6 \mathrm{~mm}$ and dust which were procured from the crusher were taken and sieve analysis was carried out to obtain individual gradation of aggregates. Desired gradation of BC mix was obtained to match the MoRTH specification as shown in table 3.5

Table 3.5: Gradation and proportioning of aggregates

\begin{tabular}{|c|c|c|c|c|c|c|c|c|c|c|c|c|}
\hline \multirow{3}{*}{$\begin{array}{c}\text { Sie } \\
\text { ve } \\
\text { siz } \\
\text { e }\end{array}$} & \multicolumn{4}{|c|}{ Obtained gradation } & \multicolumn{4}{|c|}{ Desired gradation } & \multirow{3}{*}{$\begin{array}{c}\text { Tot } \\
\text { al }\end{array}$} & \multirow{3}{*}{$\begin{array}{c}\text { Mid } \\
\text { dle } \\
\text { Limi } \\
\text { ts }\end{array}$} & \multirow{3}{*}{$\begin{array}{l}\text { Lo } \\
\text { wer } \\
\text { Lim } \\
\text { its }\end{array}$} & \multirow{3}{*}{$\begin{array}{c}\text { Up } \\
\text { per } \\
\text { Lim } \\
\text { its }\end{array}$} \\
\hline & \multirow{2}{*}{25} & \multirow{2}{*}{12} & \multirow{2}{*}{6} & \multirow{2}{*}{$\begin{array}{c}\text { Dus } \\
\mathbf{t}\end{array}$} & 25 & 12 & 6 & $\begin{array}{c}\text { Du } \\
\text { st }\end{array}$ & & & & \\
\hline & & & & & $\begin{array}{l}22 \\
\%\end{array}$ & $6 \%$ & $\begin{array}{l}32 \\
\%\end{array}$ & $\begin{array}{l}40 \\
\%\end{array}$ & & & & \\
\hline 26. & 100 & 100 & 100 & 100 & 22 & 6 & 32 & 40 & 100 & 100 & 100 & 100 \\
\hline
\end{tabular}


Study on Marshall Stability Properties of BC Mix Used In Road Construction by Adding Waste

\begin{tabular}{|c|c|c|c|c|c|c|c|c|c|c|c|c|}
\hline 19 & $\begin{array}{c}53 . \\
50\end{array}$ & 100 & 100 & 100 & $\begin{array}{l}11 . \\
77\end{array}$ & 6 & 32 & 40 & $\begin{array}{l}89 . \\
77\end{array}$ & 89.5 & 79 & 100 \\
\hline $\begin{array}{c}13 . \\
2\end{array}$ & $\begin{array}{c}4.0 \\
0\end{array}$ & $\begin{array}{l}65 . \\
50 \\
\end{array}$ & 100 & 100 & $\begin{array}{c}0.8 \\
8 \\
\end{array}$ & $\begin{array}{c}3.9 \\
3 \\
\end{array}$ & 32 & 40 & $\begin{array}{c}76 . \\
81 \\
\end{array}$ & 69 & 59 & 79 \\
\hline $\begin{array}{c}9.5 \\
0 \\
\end{array}$ & $\begin{array}{c}0.0 \\
0 \\
\end{array}$ & $\begin{array}{c}3.0 \\
0 \\
\end{array}$ & $\begin{array}{l}39 . \\
40 \\
\end{array}$ & 100 & $\begin{array}{c}0.0 \\
0\end{array}$ & $\begin{array}{c}0.1 \\
8 \\
\end{array}$ & $\begin{array}{l}12 . \\
61\end{array}$ & 40 & $\begin{array}{l}52 . \\
79 \\
\end{array}$ & 62 & 52 & 72 \\
\hline $\begin{array}{c}4.7 \\
5\end{array}$ & $\begin{array}{c}0.0 \\
0\end{array}$ & $\begin{array}{l}0.6 \\
18\end{array}$ & $\begin{array}{c}4.4 \\
0\end{array}$ & 100 & $\begin{array}{c}0.0 \\
0\end{array}$ & $\begin{array}{c}0.0 \\
4\end{array}$ & $\begin{array}{c}1.4 \\
1\end{array}$ & 40 & $\begin{array}{l}41 . \\
45\end{array}$ & 45 & 35 & 55 \\
\hline $\begin{array}{c}2.3 \\
6\end{array}$ & $\begin{array}{c}0.0 \\
0\end{array}$ & $\begin{array}{l}0.3 \\
72\end{array}$ & $\begin{array}{c}0.4 \\
61\end{array}$ & $\begin{array}{c}90.8 \\
8\end{array}$ & $\begin{array}{c}0.0 \\
0\end{array}$ & $\begin{array}{c}0.0 \\
2\end{array}$ & $\begin{array}{c}0.1 \\
5\end{array}$ & $\begin{array}{l}36 . \\
35 \\
\end{array}$ & $\begin{array}{l}36 . \\
52\end{array}$ & 36 & 28 & 44 \\
\hline $\begin{array}{c}1.1 \\
8 \\
\end{array}$ & $\begin{array}{c}0.0 \\
0 \\
\end{array}$ & $\begin{array}{l}0.3 \\
25 \\
\end{array}$ & $\begin{array}{l}0.3 \\
91 \\
\end{array}$ & $\begin{array}{c}69.6 \\
2 \\
\end{array}$ & $\begin{array}{c}0.0 \\
0 \\
\end{array}$ & $\begin{array}{c}0.0 \\
2 \\
\end{array}$ & $\begin{array}{c}0.1 \\
3 \\
\end{array}$ & $\begin{array}{l}27 . \\
85 \\
\end{array}$ & $\begin{array}{l}27 . \\
99 \\
\end{array}$ & 27 & 20 & 34 \\
\hline $\begin{array}{c}0.6 \\
0\end{array}$ & $\begin{array}{c}0.0 \\
0 \\
\end{array}$ & $\begin{array}{l}0.2 \\
79\end{array}$ & $\begin{array}{l}0.3 \\
22\end{array}$ & $\begin{array}{c}52.9 \\
2\end{array}$ & $\begin{array}{c}0.0 \\
0\end{array}$ & $\begin{array}{c}0.0 \\
2\end{array}$ & $\begin{array}{c}0.1 \\
0\end{array}$ & $\begin{array}{l}21 . \\
17 \\
\end{array}$ & $\begin{array}{l}21 . \\
29 \\
\end{array}$ & 21 & 15 & 27 \\
\hline $\begin{array}{c}0.3 \\
0 \\
\end{array}$ & $\begin{array}{c}0.0 \\
0\end{array}$ & $\begin{array}{l}0.1 \\
92 \\
\end{array}$ & $\begin{array}{l}0.1 \\
44 \\
\end{array}$ & $\begin{array}{c}36.0 \\
4\end{array}$ & $\begin{array}{c}0.0 \\
0\end{array}$ & $\begin{array}{c}0.0 \\
1 \\
\end{array}$ & $\begin{array}{c}0.0 \\
5\end{array}$ & $\begin{array}{l}14 . \\
42 \\
\end{array}$ & $\begin{array}{l}14 . \\
47 \\
\end{array}$ & 15 & 10 & 20 \\
\hline $\begin{array}{l}0.1 \\
50 \\
\end{array}$ & $\begin{array}{c}0.0 \\
0 \\
\end{array}$ & $\begin{array}{l}0.0 \\
09 \\
\end{array}$ & $\begin{array}{l}0.1 \\
09 \\
\end{array}$ & $\begin{array}{c}19.8 \\
1 \\
\end{array}$ & $\begin{array}{c}0.0 \\
0 \\
\end{array}$ & $\begin{array}{c}0.0 \\
0 \\
\end{array}$ & $\begin{array}{c}0.0 \\
3 \\
\end{array}$ & $\begin{array}{c}7.9 \\
2 \\
\end{array}$ & $\begin{array}{c}7.9 \\
6 \\
\end{array}$ & 9 & 5 & 13 \\
\hline $\begin{array}{l}0.0 \\
75\end{array}$ & $\begin{array}{c}0.0 \\
0 \\
\end{array}$ & $\begin{array}{c}0.0 \\
09\end{array}$ & $\begin{array}{c}0.1 \\
09\end{array}$ & 7.60 & $\begin{array}{c}0.0 \\
0\end{array}$ & $\begin{array}{c}0.0 \\
0\end{array}$ & $\begin{array}{c}0.0 \\
3\end{array}$ & $\begin{array}{c}3.0 \\
4 \\
\end{array}$ & $\begin{array}{c}3.0 \\
8\end{array}$ & 5 & 2 & 8 \\
\hline
\end{tabular}

Fig 3.1: Curve of BC mix with the obtained gradation for grade I

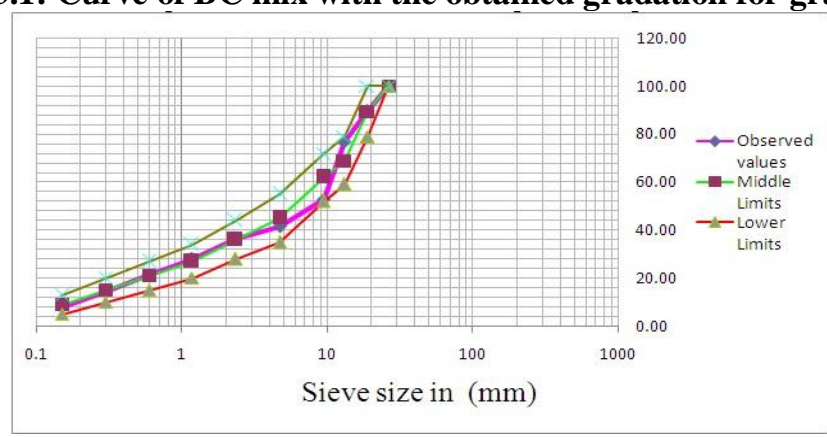

\subsection{Laboratory tests}

- Test on bitumen:

Standard tests on bitumen like penetration, softening point, ductility and flash and fire point were conducted by using appropriate apparatus.

- Tests on mixes:

Test on bituminous mixes for various properties like stability, flow value, bulk density and optimum binder content were conducted by using Marshall Stability apparatus. The properties of bituminous mix mainly depends on aggregate gradation, binder content, method of compaction and method adopted for compaction and temperature during compaction.

- Method adopted for adding plastic to the mix

Waste plastic is blended with hot bitumen at $160^{\circ} \mathrm{C}$. Powerful mechanical stirrer is required to get effective mixing. Two methods are adopted in adding plastic for bituminous mix. They are wet process and dry process

1. Wet process

Here waste plastic is mixed with bitumen and polymer modified bitumen is prepared. This polymer modified bitumen is added to hot aggregates and mixed thoroughly and is placed in Marshall Moulds.

Fig 3.2: Waste Plastics Blended With Bitumen - Aggregate Mix- Process (Wet)

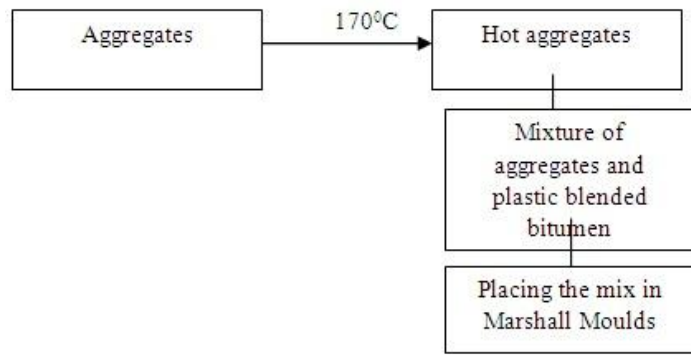


2. Dry process

In dry process plastic is added to hot aggregates and mixed thoroughly. Waste plastic gets coated on the surface of aggregates uniformly. Then bitumen is added to the polymer aggregates and mixed to get a uniform mix. This mix is placed in Marshall moulds.

Fig 3.3: Waste Plastics Blended With Bitumen - Aggregate Mix- Process (Dry)

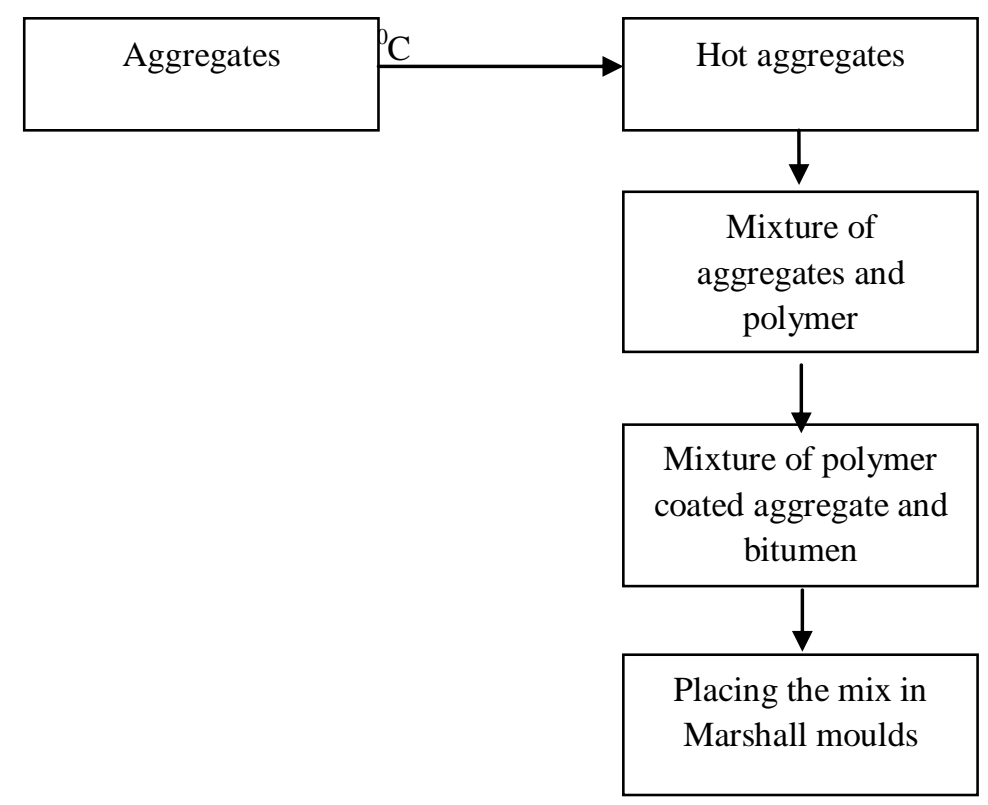

IV. Analysis of the data

Marshal moulds were prepared for different percentages of plain bitumen by varying the bitumen percentage from $3.5 \%-6.5 \%$ by increment of $0.5 \%$. The specimens were kept for $24 \mathrm{hrs}$ and then were demoulded. Marshall stability test was conducted and parameters like flow value, bulk density, percentage air voids, voids filled with bitumen (VFB) and voids filled with mineral aggregates (VMA) were calculated. The optimum bitumen content, maximum bulk density and 4\% volume of voids for bitumen grade 60/70 and 80/100 were calculated using the above properties. Also maximum stability was evaluated.

Marshall Stability method was also conducted by adding waste bottle plastic of varying percentage from $0-12 \%$ for the know binder content of both $60 / 70$ and $80 / 100$ grade bitumen. The maximum stability attained for a particular percentage of plastic is noted down.

\subsection{Parameters used for calculation:}

Percentage weight of bitumen by weight of aggregate, W4 = 3.5-6.5

Apparent specific gravity of coarse aggregate, $\mathrm{G} 1=2.72$

Apparent specific gravity of fine aggregate, $\mathrm{G} 2=2.76$

Apparent specific gravity of filler, $\mathrm{G} 3=2.5$

Apparent specific gravity of bitumen, G4

- $\quad$ Plain $80 / 100$ grade bitumen, $\mathrm{G} 4=1.017$

- $\quad$ Plain $60 / 70$ grade bitumen, $\mathrm{G} 4=1.021$

\section{.4.2 Properties of bituminous mixes}

The following properties were obtained from the laboratory studies on Bituminous Concrete

- Marshall stability value $(\mathrm{kg})$

- Flow value (mm)

- Bulk density (gm/cc)

- Percent air voids in total mix (VV)

- Voids filled with bitumen (VFB)

- Voids filled with mineral aggregates (VMA)

4.3 Results of OBC for 60/70 grade bitumen and 80/100grade bitumen

Maximum stability $=12.606 \mathrm{Kn}$, at bitumen content $=5 \%$

Maximum bulk density $=2.394 \mathrm{gm} / \mathrm{cc}$, at bitumen content $=5.5 \%$

Percent air voids $=4 \%$ at bitumen content $=5.2 \%$ 
Study on Marshall Stability Properties of BC Mix Used In Road Construction by Adding Waste

Optimum bitumen content of $60 / 70$ grade bitumen $=5.2 \%$

Maximum stability $=17.334 \mathrm{Kn}$, at bitumen content $=5 \%$

Maximum bulk density $=2.410 \mathrm{gm} / \mathrm{cc}$, at bitumen content $=5 \%$

Percent air voids $=4 \%$ at bitumen content $=4.9 \%$

Optimum bitumen content of 80/100 grade bitumen $=5 \%$

4.4 Properties of bituminous mix after adding waste plastic for 60/70 grade bitumen Table 4.1: Marshall Stability values Kg for BC Grade I for varying Waste Plastic \%

\begin{tabular}{|l|l|l|l|l|l|l|l|l|}
\hline $\begin{array}{l}\text { Waste } \\
\text { Plastic }\end{array}$ & Gt & Gb & Vv & VMA & VFB & Vb & $\begin{array}{l}\text { Stabilit } \\
\text { y } \\
\text { Value, } \\
\text { kg }\end{array}$ & $\begin{array}{l}\text { Flow } \\
\text { Value in } \\
0.25 m m\end{array}$ \\
\hline 0 & $\begin{array}{l}2.5 \\
8\end{array}$ & $\begin{array}{l}2.2 \\
84\end{array}$ & $\begin{array}{l}7.7 \\
01\end{array}$ & 19.477 & $\begin{array}{l}60.46 \\
2\end{array}$ & $\begin{array}{l}11.77 \\
6\end{array}$ & 1231 & 5.7 \\
\hline 2 & $\begin{array}{l}2.5 \\
7\end{array}$ & $\begin{array}{l}2.2 \\
85\end{array}$ & $\begin{array}{l}7.5 \\
63\end{array}$ & 19.326 & $\begin{array}{l}60.86 \\
5\end{array}$ & $\begin{array}{l}11.76 \\
3\end{array}$ & 1272 & 6 \\
\hline 4 & $\begin{array}{l}2.5 \\
4\end{array}$ & $\begin{array}{l}2.2 \\
78\end{array}$ & $\begin{array}{l}5.3 \\
43\end{array}$ & 17.201 & $\begin{array}{l}68.93 \\
7\end{array}$ & $\begin{array}{l}11.85 \\
8\end{array}$ & 1291 & 6 \\
\hline 6 & $\begin{array}{l}2.5 \\
2\end{array}$ & $\begin{array}{l}2.3 \\
56\end{array}$ & $\begin{array}{l}5.5 \\
58\end{array}$ & 17.273 & $\begin{array}{l}67.82 \\
3\end{array}$ & $\begin{array}{l}11.71 \\
5\end{array}$ & 1300 & 6 \\
\hline 8 & $\begin{array}{l}2.4 \\
8\end{array}$ & $\begin{array}{l}2.2 \\
84\end{array}$ & $\begin{array}{l}4.0 \\
67\end{array}$ & 15.786 & $\begin{array}{l}74.23 \\
8\end{array}$ & $\begin{array}{l}11.71 \\
9\end{array}$ & 1552 & 6 \\
\hline 10 & $\begin{array}{l}2.4 \\
6\end{array}$ & $\begin{array}{l}2.3 \\
27\end{array}$ & $\begin{array}{l}3.7 \\
76\end{array}$ & 15.446 & $\begin{array}{l}75.54 \\
9\end{array}$ & $\begin{array}{l}11.66 \\
8\end{array}$ & 1525 & 6 \\
\hline 12 & $\begin{array}{l}2.4 \\
3\end{array}$ & $\begin{array}{l}2.3 \\
20\end{array}$ & $\begin{array}{l}2.6 \\
58\end{array}$ & 14.283 & $\begin{array}{l}81.38 \\
7\end{array}$ & $\begin{array}{l}11.62 \\
4\end{array}$ & 1258 & 5.7 \\
\hline
\end{tabular}

Fig 4.1.1: Relation between stability and bitumen content

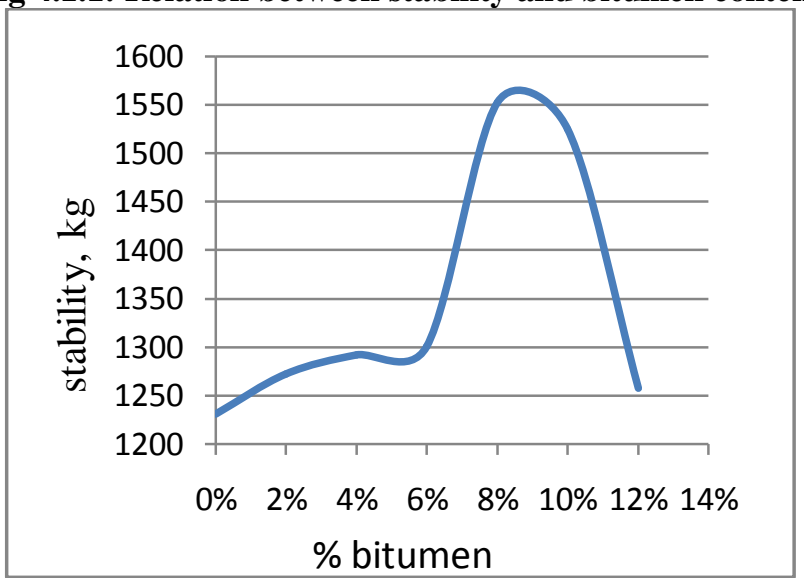

Fig 4.1.2: Relation between bulk density and bitumen content

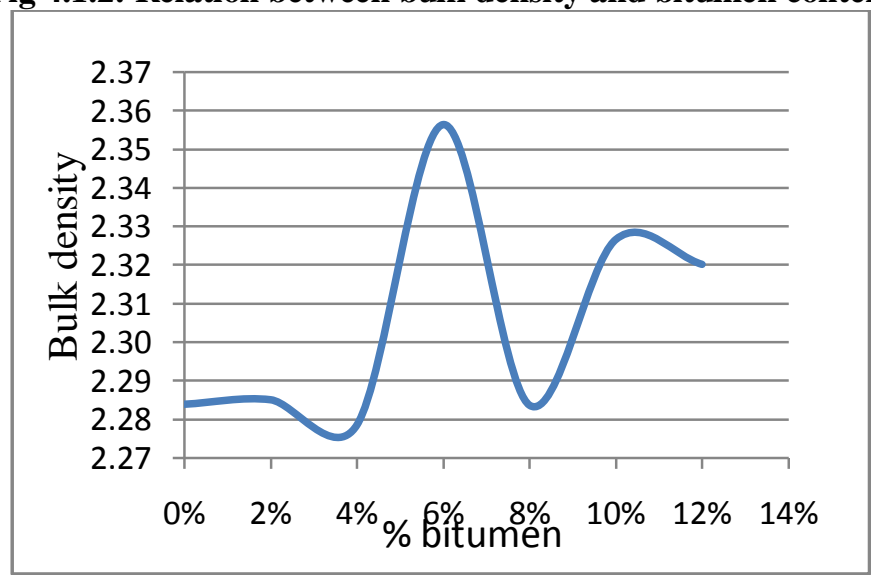


Study on Marshall Stability Properties of BC Mix Used In Road Construction by Adding Waste

Fig 4.1.3: Relation between voids filled with bitumen (VFB) and bitumen content

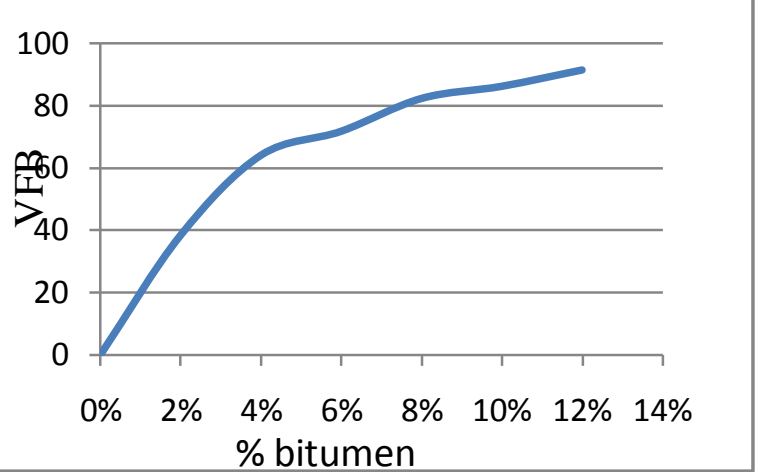

Fig 4.1.4: Relation between flow and bitumen content

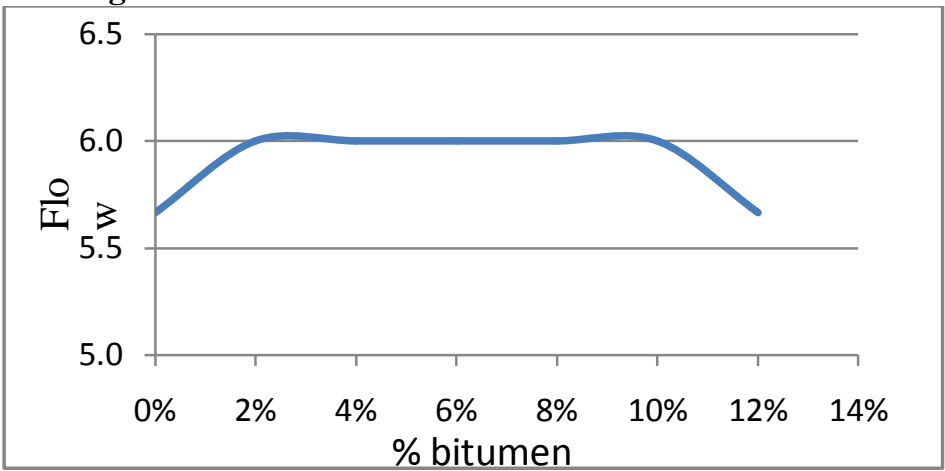

Fig 4.1.5: Relation between voids ratio ( $\mathrm{Vv}$ ) and bitumen content

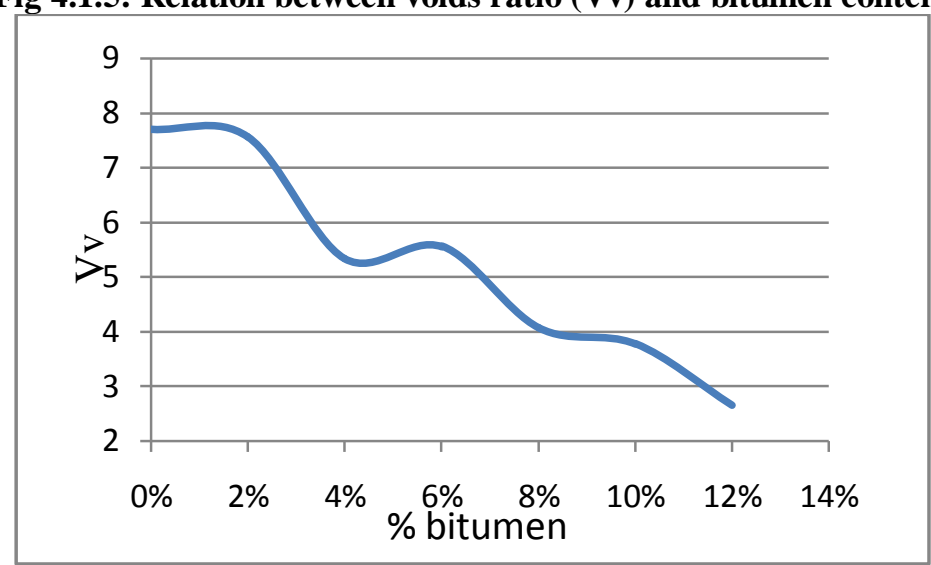

Results:

Maximum stability $=1552 \mathrm{~kg}$

Waste plastic $=\mathbf{8 \%}$ of weight of bitumen

4.5 Properties of bituminous mix after adding waste plastic for 80/100 grade bitumen

\begin{tabular}{|l|l|l|l|l|l|l|l|l|}
\hline $\begin{array}{l}\text { Waste } \\
\text { Plastic, } \\
\%\end{array}$ & Gt & Gb & Vv & VMA & VFB & Vb & $\begin{array}{l}\text { Stabilit } \\
\text { Value, } \\
\mathrm{kg}\end{array}$ & $\begin{array}{l}\text { Flow } \\
\text { Value in } \\
0.25 \mathrm{~mm}\end{array}$ \\
\hline 0 & $\begin{array}{l}2.6 \\
0\end{array}$ & $\begin{array}{l}2.33 \\
3\end{array}$ & $\begin{array}{l}11.9 \\
78\end{array}$ & $\begin{array}{l}23.61 \\
2\end{array}$ & $\begin{array}{l}49.26 \\
9\end{array}$ & $\begin{array}{l}11.63 \\
3\end{array}$ & 1529 & 5 \\
\hline 2 & $\begin{array}{l}2.5 \\
6\end{array}$ & $\begin{array}{l}2.33 \\
0\end{array}$ & $\begin{array}{l}9.13 \\
9\end{array}$ & $\begin{array}{l}20.89 \\
2\end{array}$ & $\begin{array}{l}56.25 \\
5\end{array}$ & $\begin{array}{l}11.75 \\
3\end{array}$ & 1508 & 5 \\
\hline
\end{tabular}


Study on Marshall Stability Properties of BC Mix Used In Road Construction by Adding Waste

\begin{tabular}{|l|l|l|l|l|l|l|l|l|}
\hline 4 & 2.5 & 2.34 & 7.63 & 19.43 & 60.71 & 11.80 & 1609 & 4 \\
& 3 & 4 & 6 & 6 & 1 & 0 & \\
\hline \multirow{2}{*}{6} & 2.5 & 2.32 & 6.49 & $\begin{array}{l}18.29 \\
5 \\
5\end{array}$ & $\begin{array}{l}64.51 \\
8\end{array}$ & $\begin{array}{l}11.80 \\
3\end{array}$ & 1672 & 4.3 \\
\hline 8 & $\begin{array}{l}2.4 \\
9\end{array}$ & $\begin{array}{l}2.39 \\
4\end{array}$ & $\begin{array}{l}4.65 \\
2\end{array}$ & $\begin{array}{l}16.58 \\
0\end{array}$ & $\begin{array}{l}71.94 \\
2\end{array}$ & $\begin{array}{l}11.92 \\
8\end{array}$ & 1963 & 4.7 \\
\hline \multirow{2}{*}{10} & $\begin{array}{l}2.4 \\
6\end{array}$ & $\begin{array}{l}2.32 \\
6\end{array}$ & $\begin{array}{l}5.84 \\
2\end{array}$ & $\begin{array}{l}17.50 \\
5\end{array}$ & $\begin{array}{l}66.62 \\
7\end{array}$ & $\begin{array}{l}11.66 \\
3\end{array}$ & 1736 & 4.7 \\
\hline \multirow{2}{*}{12} & $\begin{array}{l}2.4 \\
0\end{array}$ & $\begin{array}{l}2.31 \\
5\end{array}$ & $\begin{array}{l}2.14 \\
0\end{array}$ & $\begin{array}{l}13.92 \\
5\end{array}$ & $\begin{array}{l}84.63 \\
1\end{array}$ & $\begin{array}{l}11.78 \\
5\end{array}$ & 1573 & 4 \\
\hline
\end{tabular}

Table 4.2: Marshall Stability values Kg for BC Grade I for varying Waste Plastic \%

Fig 4.2.1: Relation between stability and bitumen content

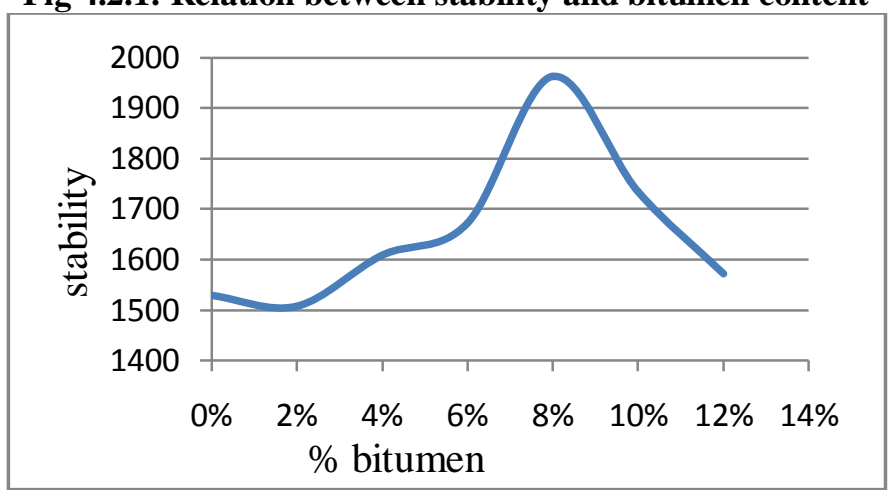

Fig 4.2.2: Relation between bulk density and bitumen content

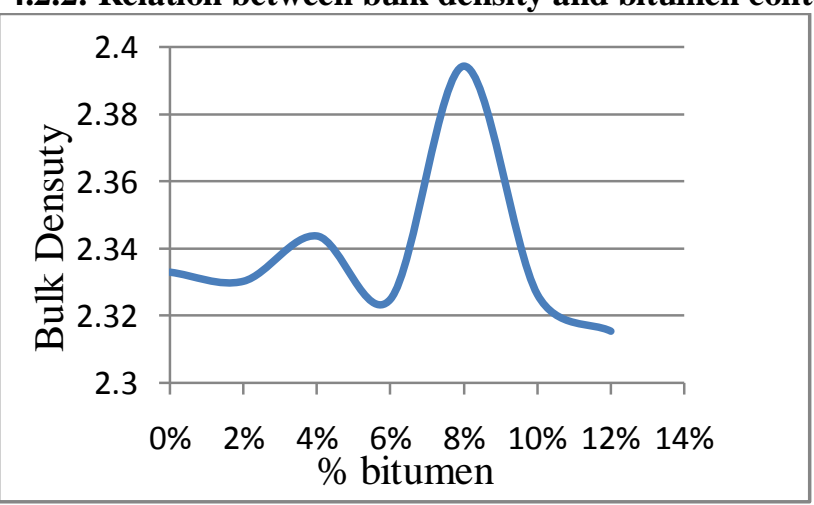

Fig 4.2.3: Relation between voids filled with bitumen (VFB) and bitumen content

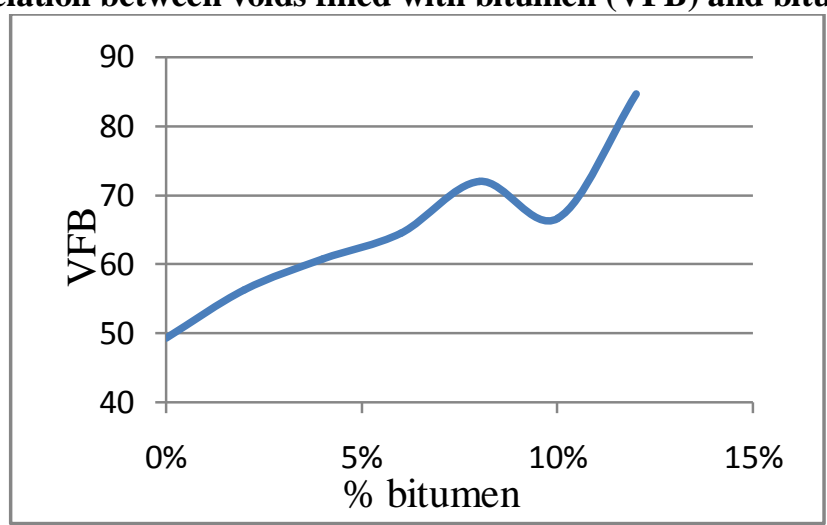


Study on Marshall Stability Properties of BC Mix Used In Road Construction by Adding Waste

Fig 4.2.4: Relation between voids ratio $(\mathrm{Vv})$ and bitumen content

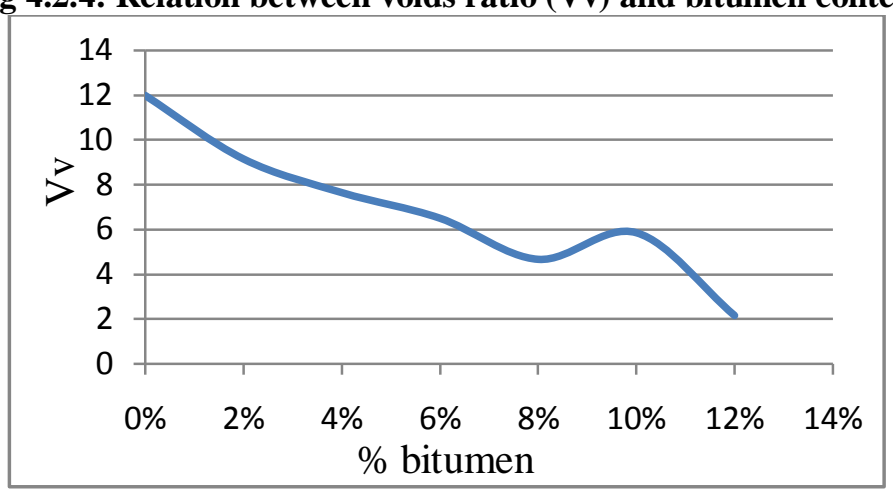

Fig 4.2.5: Relation between flow and bitumen content

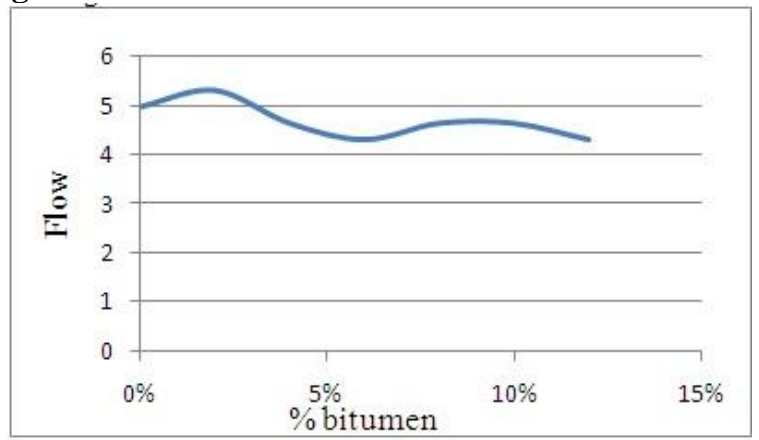

Result:

Maximum stability $=1963 \mathrm{~kg}$

Waste plastic $=\mathbf{8 \%}$ of weight of bitumen

4.6 Comparison of Marshall Stability values $\mathrm{Kg}$ for BC grade I for varying plastic percentage between $60 / 70$ and $80 / 100$ grade bitumen

Table 4.3: Comparison of Marshall Stability values $\mathbf{b} / \mathbf{n} 60 / 70$ and 80/100 grade bitumen

\begin{tabular}{|l|l|l|l|}
\hline \multirow{2}{*}{ Waste plastic \% } & GRADE I & \\
\cline { 2 - 4 } & $\begin{array}{l}\text { Wp coated with aggregates } \\
60 / 70\end{array}$ & $\begin{array}{l}\text { Wp coated with aggregates } \\
80 / 100\end{array}$ \\
\hline $0 \%$ & 1231 & 1529 \\
\hline $2 \%$ & 1272 & 1508 \\
\hline $4 \%$ & 1291 & 1609 \\
\hline $6 \%$ & 1300 & 1672 \\
\hline $8 \%$ & 1552 & 1963 \\
\hline $10 \%$ & 1525 & 1736 \\
\hline $12 \%$ & 1258 & 1573 \\
\hline
\end{tabular}

Fig 4.3.1: Comparison of stability values $b / n$ 60/70 and 80/100

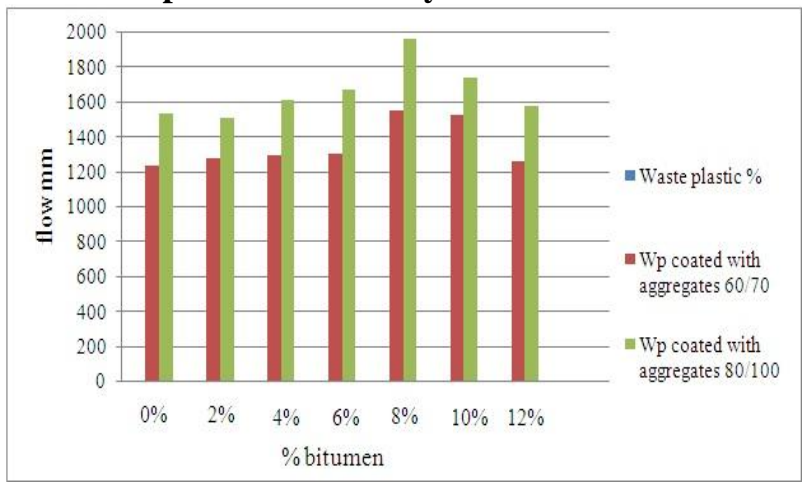


Study on Marshall Stability Properties of BC Mix Used In Road Construction by Adding Waste

From the above graph the maximum stability is attained in 80/100 grade bitumen as that of 60/70 grade. Stability attained in $80 / 100$ grade bitumen is $1963 \mathrm{~kg}$ and in 60/70 grade bitumen is $1552 \mathrm{~kg}$. the stability value obtained is $20 \%$ more in $80 / 100$ grade bitumen as compared with $60 / 70$ grade.

4.7 Comparison of Flow values for BC grade I for varying plastic percentage between 60/70 and 80/100 grade bitumen

Table 4.4: Comparison of Flow Values B/N 60/70 and 80/100 Grade Bitumen

\begin{tabular}{|l|l|l|}
\hline \multirow{3}{*}{ Waste plastic \% } & GRADE I \\
\cline { 2 - 3 } & $\begin{array}{l}\text { Wp coated with aggregates } \\
60 / 70\end{array}$ & $\begin{array}{l}\text { Wp coated with aggregates } \\
80 / 100\end{array}$ \\
\hline $0 \%$ & 5.7 & 5 \\
\hline $2 \%$ & 6 & 5 \\
\hline $4 \%$ & 6 & 4.7 \\
\hline $6 \%$ & 6 & 4.3 \\
\hline $8 \%$ & 6 & 4.7 \\
\hline $10 \%$ & 6 & 4.7 \\
\hline $12 \%$ & 5.7 & 4.3 \\
\hline
\end{tabular}

Fig 4.4.1: Comparison of floe $b / n$ 60/70 and 80/100 grade bitumen

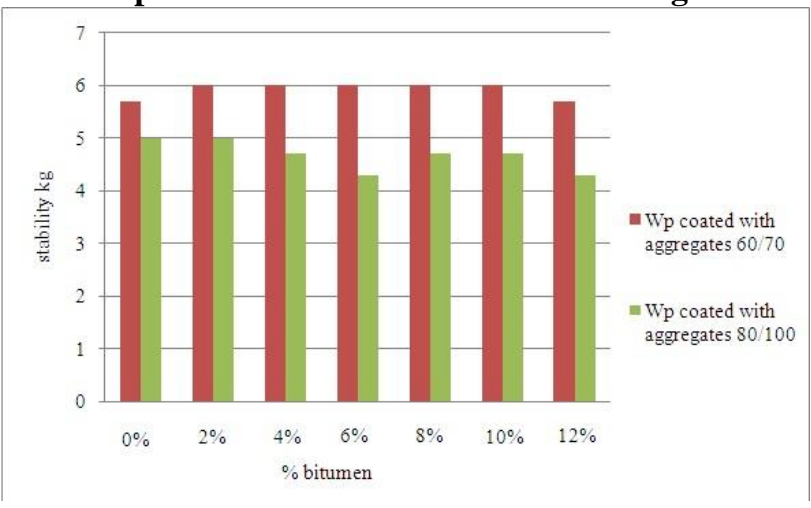

The flow value remains unchanged in 60/70 grade bitumen. But the flow value has been continuosly varying in the $80 / 100$ grade bitumen.

4.8 Comparison between stability values of soaked and unsoaked specimens of $60 / 70$ and 80/100 grade bitumen

Table 4.5: Comparison of Marshall Stability Test Results, For 1, 2 and 3days Soaked and Unsoaked Values

\begin{tabular}{|l|l|l|l|l|}
\hline \multirow{2}{*}{ Type of plastic blended } & \multicolumn{4}{|l|}{$8 \%$ waste plastic @ 5.2\% and 5\% bitumen } \\
\cline { 2 - 5 } & Unsoaked & $\begin{array}{l}\text { 1day } \\
\text { Soaked }\end{array}$ & 2day soaked & 3day soaked \\
\cline { 2 - 5 } & BC Grade I & \multicolumn{4}{l|}{} \\
\hline $\begin{array}{l}\text { OBC of 60/70 grade } \\
\text { bitumen }\end{array}$ & 1285 & 1217 & 1079 & 982 \\
\hline $\begin{array}{l}\text { Waste plastic coated } \\
\text { with aggregate 60/70 }\end{array}$ & 1552 & 1369 & 1357 & 1265 \\
\hline $\begin{array}{l}\text { OBC of 80/100 grade } \\
\text { bitumen }\end{array}$ & 1767 & 1419 & 1322 & 1284 \\
\hline $\begin{array}{l}\text { Waste plastic coated } \\
\text { with aggregate 80/100 }\end{array}$ & 1963 & 1813 & 1426 & 1284 \\
\hline
\end{tabular}


Fig 4.5.1: Comparison of soaked and unsoaked test results
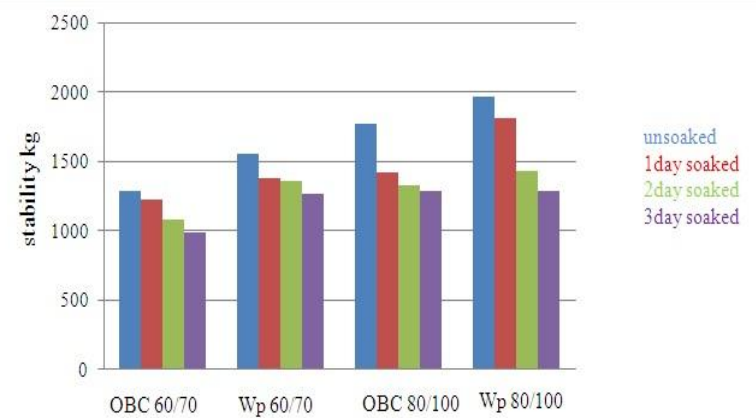

From the above graph we can see that there is a decrease in stability value. Unsoaked specimens show higher results than soaked specimen. The soaked specimens of 3days show the least stability values.

\section{$5.1 \quad$ General}

\section{Discussions and conclusions}

Use of waste plastic has made a good progress in bituminous road construction in recent years. Waste plastic are used in bituminous courses Viz BM BC SDBC PMC and MSS. This investigation is on attempt to evaluate the addition of waste plastic bottles to bituminous concrete (BC) wearing course mix of aggregate gradation I along with plain 60/70 and 80/100 bitumen.

\subsection{Optimum bitumen content for bituminous concrete (BC) mix}

Optimum bitumen content obtained for bituminous concrete grade I mix for 60/70 grade bitumen was $5 \%$ and $80 / 100$ grade bitumen was $5.1 \%$ as per the specification of MORT\&H standards. The stability obtained for the respective $\mathrm{OBC}$ was $12.606 \mathrm{Kn}$ and $17.334 \mathrm{Kn}$.

\subsection{Addition of waste plastic bottles to bituminous concrete mix} concrete mix.

Waste shredded plastic bottle were added in the increasing percentage of $0 \%-12 \%$ to bituminous

\subsection{Effect of method of coating on the mix parameters of BC grade I mix} in following:

Addition of $2 \%$ to $12 \%$ waste shredded plastic bottles by the weight of bitumen to $\mathrm{BC}$ mix has resulted

\section{i. For plain 60/70 bitumen}

a) The waste plastic which is added to aggregate mix by heating upto $260-280^{\circ} \mathrm{C}$. then the bitumen is added to form the grade I bituminous concrete and optimum bitumen content of $5 \%$ has shown the following results.

- The maximum stability was $1552 \mathrm{Kg}$ at $8 \%$ waste plastic by the weight of bitumen, $6 \mathrm{~mm}$ flow at $8 \%$ waste plastic and $74.238 \mathrm{VFB}$ at $8 \%$ waste plastic

- Bulk density $\Upsilon \mathrm{b}$ was found to be maximum of $2.356 \mathrm{gm} / \mathrm{cc}$ at $6 \%$ waste plastic and then reduces to 2.284 $\mathrm{gm} / \mathrm{cc}$ at $8 \%$ waste plastic.

- Voids in the total mix $\mathrm{Vv}$ varies from $4 \%$ to $10 \%$ by varying the waste plastic content from $2 \%-12 \%$ and at $8 \%$ waste plastic $\mathrm{Vv}$ was found to be $4.067 \%$.

b) By blending shredded waste plastic to the bitumen by heating and then adding the required aggregate to form $\mathrm{BC}$ mix grade I resulted in improper mix and stability obtained is less than that of the optimum binder content $[\mathrm{OBC}]$. This is due to following,

- Improper blending of plastic in bitumen. This is because the melting point of poly ethylene teraphthalate $[\mathrm{PET}]$ is $260-280^{\circ} \mathrm{C}$.

- Since the melting temperature of plastic is too high it is difficult to melt plastic in bitumen to get a proper blend.

- Bitumen should be heated up to the temperature of $260-280^{\circ} \mathrm{C}$ in order to obtain the proper blend. If this is done there will be a chance of bitumen to catch fire and also there will be a loss in weight of bitumen.

ii. For plain $80 / 100$ bitumen

a) The waste plastic which is added to aggregate mix by heating upto $260-280^{\circ} \mathrm{C}$. Then the bitumen is added to form the grade I bituminous concrete and optimum bitumen content of $5 \%$ has shown the following results.

- The maximum stability was $1963 \mathrm{Kg}$ at $8 \%$ waste plastic by the weight of bitumen, $4.7 \mathrm{~mm}$ flow at $8 \%$ waste plastic and $71.942 \mathrm{VFB}$ at $8 \%$ waste plastic

- Bulk density $\gamma \mathrm{b}$ was found to be maximum of $2.394 \mathrm{gm} / \mathrm{cc}$ at $8 \%$ waste plastic and then reduces to 2.315 $\mathrm{gm} / \mathrm{cc}$ at $12 \%$ waste plastic. 
- Voids in the total mix Vv varies from $8 \%$ to $10 \%$ by varying the waste plastic content from $2 \%-12 \%$ and at $8 \%$ waste plastic $\mathrm{Vv}$ was found to be $4.652 \%$.

b) By blending shredded waste plastic to the bitumen by heating and then adding the required aggregate to form $\mathrm{BC}$ mix grade I resulted in improper mix and stability obtained is less than that of the optimum binder content $[\mathrm{OBC}]$. This is due to following,

- Improper blending of plastic in bitumen. This is because the melting point of poly ethylene teraphthalate [PET] is $260-280^{\circ} \mathrm{C}$.

- Since the melting temperature of plastic is too high it is difficult to melt plastic in bitumen to get a proper blend.

- Bitumen should be heated up to the temperature of $260-280^{\circ} \mathrm{C}$ in order to obtain the proper blend. If this is done there will be a chance of bitumen to catch fire and also there will be a loss in weight of bitumen.

All the parameters like stability, flow, bulk density, voids and VFB shows that, the addition of waste plastic in mix has no much change in fluidity and rigidity parameters as compared to that of plain bituminous mix.

Stability of $80 / 100$ bitumen at $8 \%$ of waste plastic coated on aggregates has shown higher value than that of the $60 / 70$ grade bitumen.

\subsection{Water sensitivity test results}

The water sensitivity measured in terms of the Marshall stability shows the the following results'

- The stability value of the mix in unsoaked condition has high values than compared to the soaked specimens

- Stability value decreases as the soaking time of the specimen increases. Specimen with 3days soaking results in least stability than that of 1day and 2day soaking periods.

\section{Conclusions}

In the present study, the importance was to add the shredded waste plastic bottles to bituminous concrete (BC) mix and to evaluate the various mix properties like Marshall Stability, flow, bulk density, voids in the mix and VFB. Also the effect of soaking conditions of the mix was investigated. Indirect tensile strength was investigated for $\mathrm{OBC}$ and $8 \%$ plastic coated on aggregates which had yielded the highest marshal stability.

- The optimum plastic content for $60 / 70$ and $80 / 100$ grade bitumen was $8 \%$.

- For both $60 / 70$ and $80 / 100$ grade bitumen with plastic content $8 \%$, the maximum stability was achieved in $80 / 100$ grade bitumen.

- Wet process i.e. blending of plastic and bitumen cannot be carried out due to the plastic which is used has a very high melting point.

- There is an increase in stability up to $15 \%$ and $10 \%$ after adding waste plastic to the mix in $60 / 70$ and $80 / 100$ grade bitumen respectively.

- There is a decrease in stability value in water sensitivity test results. Unsoaked specimens show high stability value but soaked specimens showed a decreasing stability value.

Hence there is an increase in stability with the addition of PET plastic in asphalt mix by incorporating dry process this can be used in highway construction for better stability for the appropriate traffic.

\section{References}

[1]. Mahabir Panda and Mayajit Mazumdar(1999). "Engineering Properties of EVA-Modified Bitumen For Paving Mixes". Journal Of Materials In Civil Engineering ASCE / May 1999

[2]. Romona, Ledesma and Isacc8 L. L. "Effect of Poly Ethylene Teraphthalate from Plastic Waste on the Thermal Properties of Asphalt". The City College of Cuny Dept. of Chemical Engineering New York.

[3]. Hartman A M, Gilchrist M D and Walsh G (2001). "Effect of Mixture Compaction on Indirect Tensile Stiffness and Fatigu e". Journal Of Transportation Engineering Sep/Oct 2001

[4]. Punith V.S and Veeraraghavan A (2007). "Behavior of Asphalt Concrete Mixtures with Reclaimed Polyethylene as Additive". Journal Of Materials In Civil Engineering ASCE / June 2007 / 501

[5]. Lee Zhen Hao (2009). "Performance Characteristics of Hot Mix Asphalt with Recycled Materials". Bachelor of Engineering Report, School of Civil and Environmental Engineering, Nayang Technological University Singapore.

[6]. Sabina, Tabrez A Khan, Sangita, Sharma D K and Sharma B M (2009). "Performance Evaluation Of Waste Plastic/Polymer Modified Bituminous Concrete Mixes". Journal of scientific and industrial research Vol.68, November 2009, pp. 975-979.

[7]. Shivani Gupta and Veeraragavan A (2009). "Fatigue behavior of polymer modifies bituminous concrete mixtures". Journal of Indian road congress January-March 2009.

[8]. Zahra Niloofar Kalantar, Abdelaziz Mahrez and Rehan karim (2010). "Properties of bituminous binder modified with waste polyethylene teraphthalate". Proceedings Of Malaysian Universities Transportation Research Forum And Conferences 2010 (MUTHRFC 2010) 21 Dec 2010.

[9]. Khanna S K, Justo C E G and Veeraragavan A (2000). "Highway Material And Pavement Testing (Labouratory Manual)", Nemchand and Bros, Roorkee 2000.

[10]. Ministry of Road Transport and Highways (2001). "Specifications for road and bridge works" Fourth Revision, Published by Indian Road Congress on behalf of the Government of India. 